This is a postprint version of the following published document:

Proceedings of the Combustion Institute 35 (2015) pp. 3463-3470 DOI: $10.1016 /$ j.proci.2014.05.075

(C) 2014 The Combustion Institute. Published by Elsevier Inc

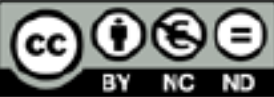

This work is licensed under a Creative Commons Attribution-NonCommercialNoDerivatives 4.0 International License. 


\title{
Effect of an external electric field on the propagation velocity of premixed flames
}

\author{
Mario Sánchez-Sanz ${ }^{a}$, Daniel C. Murphy ${ }^{\text {b,* }}$, C. Fernandez-Pello ${ }^{\text {b }}$ \\ a Dept. Ingeniería Térmica y de Fluidos, Universidad Carlos III de Madrid, 28911 Leganés, Spain \\ ${ }^{\mathrm{b}}$ Department of Mechanical Engineering, University of California, Berkeley, United States
}

\begin{abstract}
There have been many experimental investigations into the ability of electric fields to enhance combustion by acting upon ion species present in flames [1]. In this work, we examine this phenomenon using a one-dimensional model of a lean premixed flame under the influence of a longitudinal electric field. We expand upon prior two-step chain-branching reaction laminar models with reactions to model the creation and consumption of both a positively-charged radical species and free electrons. Also included are the electromotive force in the conservation equation for ion species and the electrostatic form of the Maxwell equations in order to resolve ion transport by externally applied and internally induced electric fields. The numerical solution of these equations allows us to compute changes in flame speed due to electric fields. Further, the variation of key kinetic and transport parameters modifies the electrical sensitivity of the flame. From changes in flame speed and reactant profiles we are able to gain novel, valuable insight into how and why combustion can be controlled by electric fields.
\end{abstract}

Keywords: Premixed flame; Electric field; Laminar flame speed; Charged species; Chain-branching reaction

\section{Introduction}

In order to study the effect of the electric field on a premixed flame, this paper will consider a one-dimensional model of a lean premixed flame under the influence of a longitudinal electric field.

\footnotetext{
* Corresponding author. Address: 60 Hesse Hall, Department of Mechanical Engineering, University of California, Berkeley, United States. Fax: +1 (510) 642 1850 .

E-mail addresses: mssanz@ing.uc3m.es (M. SánchezSanz), dmurphy0516@gmail.com (D.C. Murphy), ferpello@me.berkeley.edu (C. Fernandez-Pello).
}

The electric field is oriented in the direction of the gas flow and can have positive or negative signs, indicating different electric field polarities. The model is defined by the set of conservation equations and chemical reactions which should reflect the behavior of a lean premixed flame subjected to an electric field. To model the interaction between the flame and the electric field, we need to include in the chemistry model a radical that can be ionized at high temperatures to give a protons and electrons that can be affected by the electric field.

A good candidate is the the two-step, chainbranching chemistry model developed originally by $[2,3]$ and further developed later by [4] using 


\begin{tabular}{|c|c|c|c|}
\hline \multicolumn{4}{|c|}{ Nomenclature } \\
\hline$v$ & mobility & $\mathcal{A}$ & dimensionless frequency factor for \\
\hline$\rho$ & mixture density & & reaction III \\
\hline$A$ & frequency factor & $\mathcal{B}$ & dimensionless frequency factor for \\
\hline$c$ & concentration & & reaction IV \\
\hline $\begin{array}{l}c_{p} \\
D\end{array}$ & $\begin{array}{l}\text { heat capacity } \\
\text { diffusivity }\end{array}$ & $\Delta \beta$ & $\begin{array}{l}\text { dimensionless activation energy } \\
\text { increment }\end{array}$ \\
\hline$e$ & elementary charge & $\gamma$ & dimensionless heat release \\
\hline$E_{a}$ & activation energy & $\mu$ & eigenvalue of dimensionless conserva- \\
\hline$m$ & mass of particle & & tion equations \\
\hline$R_{g}$ & gas constant & $k$ & iteration counter \\
\hline$S_{L}^{\circ}$ & Flame Speed & Le & Lewis number \\
\hline$V$ & diffusion velocity & & \\
\hline$W$ & molecular weight & \multirow{2}{*}{\multicolumn{2}{|c|}{ Subscripts }} \\
\hline Z & charge & & \\
\hline$\Omega[\omega]$ & reaction rate [dimensionless] & 0 & initial or imposed value \\
\hline$\varepsilon_{0}[\varepsilon]$ & vacuum permittivity [dimensionless] & $\infty$ & final value \\
\hline$E^{\prime}[E]$ & electric field [dimensionless] & $e^{-}$ & electron \\
\hline$q[Q]$ & heat of reaction [dimensionless] & $F$ & fuel \\
\hline$T[\theta]$ & temperature [dimensionless] & $T$ & temperature, thermal \\
\hline$x^{\prime}[x]$ & spatial coordinate [dimensionless] & $\begin{array}{l}Z \\
7^{+}\end{array}$ & neutral radical \\
\hline$Y^{\prime}[Y]$ & mass fraction [dimensionless] & & positive radical \\
\hline$\alpha$ & relaxation parameter & & \\
\hline$\beta$ & Zeldovich number & & \\
\hline
\end{tabular}

the high activation energy asymptotic. The model was later slightly modified in $[5,6]$ by linearizing the radical recombination step, allowing the definition of an explicit crossover temperature below which the chain-branching reaction remains frozen. Some authors refer this two-step kinetic model as a better approach to real hydrocarbons and hydrogen flames description than the one-step model [5-7]. Chain-branching reactions are typically located in the high-temperature region of the flame because of its high activation energy. These reactions produce an increase of the intermediate species or chemical radicals which diffuse upstream and downstream of this thin chain-branching zone and recombine by means of a very exothermic chain-termination reaction in a wider region. Therefore, the heat release occurs throughout the flame and fuel exhaustion is reached interior to the flame [6,7], a real characteristic of flames. In the one-step model fuel consumption and final adiabatic flame is reached all at once.

To complement the model proposed by Dold, we incorporate here two additional reactions to account for the production and consumption of charged species. According to [8], the source of ions is generally accepted to be the chemiionization reaction in which a radical reacts with a third body to give a proton and an electron. Later, the proton would recombine with the electrons via a dissociative recombination to release a certain amount of heat. When an external electric field is imposed across the flame front, the charged particles are removed from the reaction zone at a rate proportional to the electric field strength until the field strength is large enough to make the removal rate of electrons and protons equal to the chemiionization formation rate, leading to current saturation.

Table 1 shows the reactions used to model the effect of the electric field in a premixed flame. As we anticipated above, reactions I and II replicate the model proposed by Dold while reaction III and IV model the chemiionization and the dissociative recombination, respectively, first proposed by $[9,10]$ and used in multiple numerical studies $[8,11,12]$.

\section{Formulation}

Consider a planar premixed flame propagating with a constant velocity, $S_{L}$, with respect to an unburned gas at initial temperature $T_{0}$ and fuel mass fraction $Y_{F_{0}}$. The mixture is assumed to be deficient in fuel and the mass fraction of the oxidizer, which is in abundance, remains nearly constant. For the sake of simplicity, the paper deals with a diffusive-thermal model, according to which $\rho, c_{p}, D_{T}, D_{F}, D_{Z}$ are all constant.

The equations describing the structure of this flame in the presence of an electric field include 
Table 1

Chain-branching, chemionizaition and dissociative recombination reactions used in this formulation.

\begin{tabular}{lll}
\hline$i_{\mathrm{SD}}$ & Reaction & \\
\hline $\mathrm{I}$ & $\mathrm{F}+\mathrm{Z} \rightarrow 2 \mathrm{Z}$ & $\Omega_{I}=A_{I} \frac{\rho^{2}}{W_{F} W_{Z}} Y_{Z}^{\prime} Y_{F}^{\prime} \exp \left(-E_{I} / R T\right)$ \\
II & $\Omega_{I I}=A_{I I} \frac{\rho^{2}}{W_{Z} \bar{W}} Y_{Z}^{\prime}$ \\
III & $\mathrm{Z}+\mathrm{M} \rightarrow \mathrm{P}+\mathrm{M}+\mathrm{q}_{\mathrm{II}}$ & $\Omega_{I I I}=A_{I I I} \frac{\rho^{2}}{W_{Z} \bar{W}} Y_{Z}^{\prime} \exp \left(-E_{I I I} / R T\right)$ \\
IV & $\mathrm{Z}+\mathrm{M} \rightarrow \mathrm{Z}^{+}+\mathrm{e}^{-}+\mathrm{M}$ & $\Omega_{I V}=A_{I V} \frac{\rho^{2}}{W_{Z^{+}} W_{e^{-}}} Y_{Z^{+}}^{\prime} Y_{e}^{\prime}$ \\
\hline
\end{tabular}

the mass and species conservation equations. Additionally, the model needs to consider the effect that the electric field exerts on the charged species. Since the concentrations of charged species are usually small, we will assume that the relation defining the flux of the $i$ th species due to diffusion plus electromigration will be that for a weakly-ionized plasma, an extreme that considerably simplifies our treatment of the problem and which is usually the case in real flames [13]. Thus, the contributions to the flux of charged species can be linearly superposed to define the Nerst-Planck equation describing the interaction between the electric field and the charged species [14].

With currents and electric fields present, the laws of electrodynamics should be incorporated, in the form of the Maxwell equations, into the equations of mass and energy conservation. Nevertheless, if there are no magnetic fields and the electric field does not change with time, the electrodynamic problem reduces to an electrostatic one. In this case, the electric field can be computed as $\partial E^{\prime} / \partial_{x}=\left(c_{+}-c_{-}\right) e / \varepsilon_{0}$

In an electrically neutral ionized gas, the condition $\sum c_{i} Z_{i} \simeq 0$ is satisfied, where $Z_{i}$ and $c_{i}$ are the charge and the concentration of the particle $i$, respectively. Since the electrons represent nearly $90 \%$ of the negative charge carriers [15], we can write the electrons-to-protons characteristic mass fraction ratio as $Y_{e^{-}, 0} / Y_{Z^{+}, 0} \simeq m^{-} / m^{+} \ll 1$, indicating that the contribution of the electrons to the total mass of the gas is small.

In the constant density approximation we are considering here, the problem reduces to the integration of energy and mass transport equations for neutral and charged species. Unlike neutral gases, where diffusion is controlled by Fick's law, the presence of an electric field can change the way in which the particles are redistributed in an inhomogeneous mixture. Even when no external electric field is applied, a displacement of charged particles would create a charge imbalance that, in turn, would induce an electric field opposing the charge displacement. This effect can be taken into account by defining the diffusion velocity $V^{k}$ as $[8,14]$ $\rho Y V_{j}^{k}=-\rho D_{j} \frac{\partial Y_{i}}{\partial x}+Z_{k} \rho v^{k} Y_{i} E$,

where $Z_{k}$ is negative if the species is negatively charged, positive if the species is positively charged and zero if the species is neutral. The diffusion coefficient of the neutral species is considered constant, being $D_{F}$ and $D_{Z}$ the diffusion coefficients of fuel and radical $Z$, respectively. On the other hand, Belhi et al. [12] introduced, following Delcroix [16], the following expression for the ratio between the diffusion coefficient of electrons and ions $D_{e^{-}}=D_{Z^{+}}\left(m^{+} / m^{-}\right)^{1 / 2}$ where $m^{+}$and $m^{-}$are the mass of a single proton and electron respectively.

The mobility of a charged particles $v_{i}$ is defined as the ratio between its drift velocity and the electric field strength. The strict calculation of the mobility of ions $v_{Z^{+}}$and electrons $v_{e^{-}}$would imply the appropriate assessment of the effect of the temperature and concentration changes on $v_{i}$ [11]. Nevertheless, and for the sake of simplicity, we will assume hereafter constant proton and electron mobilities. Furthermore, we can write the ratio between the mobilities of electrons and ions by using the Einstein relationship given in [12] $v_{e^{-}}=v_{Z^{+}}\left(m^{+} / m^{-}\right)^{1 / 2}$.

The four-step, chain-branching kinetic mechanism used here to model the effect of the electric field includes the autocatalytic and recombination steps given above in Table 1 , where $\Omega_{I}$ is the temperature-sensitive, chain-branching reaction rate, with $E_{I}$ the activation energy and $A_{I}$ the frequency factor, and $\Omega_{I I}$ is the temperature-independent completion reaction rate, with $A_{I I}$ the rate constant. Notice that, as indicated by [6], the reaction can only take place if the temperature $T>T_{c}$, where $T_{c}$ is the branching temperature that takes into account the amount of radical removed by diffusion from the inner branching zone and is obtained by imposing $\Omega_{I}=\beta^{2} \Omega_{I I}$ to give

$\frac{A_{I}}{A_{I I}} \frac{W}{W_{F}} Y_{F_{0}}=\left\{\frac{E}{R_{g}} \frac{T_{c}-T_{0}}{T_{c}}\right\}^{2} e^{E_{I} / R_{g} T_{c}}$

This temperature is used here to define the nondimensional temperature $\theta=\left(T-T_{0}\right) /\left(T_{c}-T_{0}\right)$ and the Zel'dovich number $\beta=\left(T_{c}-T_{0}\right) /\left(R_{g} T_{0}^{2}\right)$. 
A great amount of work has been done to identify the ions species present in a flame and the mechanisms responsible for their production. A detailed account can be found in [8-10]. In this regard we incorporate in our simplified model the steps III and IV that account for the temperaturedependent chemiionization of the radical $Z$ and the posterior exothermic recombination of protons and electrons.

As a summary of the exposed above, we introduce in the energy and mass conservation equations the non-dimensional temperature $\theta$ and spatial coordinate $x=x^{\prime} /\left(D_{T} / S_{L}\right)$ and the scaled mass fractions of fuel $Y_{F}=Y_{F}^{\prime} / Y_{F_{0}}$, radical $Y_{Z}=$ $Y_{Z}^{\prime} /\left(W_{Z}\left(Y_{F_{0}} / W_{F}\right)\right.$, protons $Y_{Z^{+}}=Y_{Z^{+}}^{\prime} /\left(Y_{F_{0}}\right.$ $\left.\left(W_{Z} / W_{F}\right)\right)$ and electrons $Y_{e^{-}}=Y_{e^{-}}^{\prime} /\left(Y_{F_{0}}\left(\mathrm{~m}^{-} / \mathrm{m}^{+}\right)\right.$ $\left.\left(W_{Z} / W_{F}\right)\right)$ to yield the non-dimensional conservation equations

$\frac{d \theta}{d x}=\frac{d^{2} \theta}{d x^{2}}+\mu Q\left(\omega_{I I}+\omega_{I V} \frac{q_{I V}}{q_{I I}}\right)$

$\frac{d Y_{F}}{d x}=\frac{1}{L e_{F}} \frac{d^{2} Y_{F}}{d x^{2}}-\mu \omega_{I}$

$\frac{d Y_{Z}}{d x}=\frac{1}{L e_{Z}} \frac{d^{2} Y_{Z}}{d x^{2}}+\mu\left[\omega_{I}-\omega_{I I}-\omega_{I I I}\right]$

$\frac{d Y_{Z^{+}}}{d x}=-\mu^{1 / 2} \frac{d\left(E Y_{Z^{+}}\right)}{d x}+\frac{1}{L e_{Z^{+}}} \frac{d^{2} Y_{Z^{+}}}{d x^{2}}$

$$
+\mu\left[\omega_{I I I}-\omega_{I V}\right]
$$

$\frac{d Y_{e^{-}}}{d x}=\mu^{1 / 2}\left(\frac{m^{+}}{m^{-}}\right)^{1 / 2} \frac{d\left(E Y_{e^{-}}\right)}{d x}+\left(\frac{m^{+}}{m^{-}}\right)^{1 / 2} \frac{1}{L e_{Z^{+}}} \frac{d^{2} Y_{e^{-}}}{d x^{2}}$ $+\mu\left[\omega_{I I I}-\omega_{I V}\right]$

with boundary conditions $\theta=Y_{Z}=Y_{Z^{+}}=$ $Y_{e^{-}}=Y_{F}-1=0$ at $x \rightarrow-\infty$ and $\theta^{\prime}=Y_{F}^{\prime}=$ $Y_{Z}^{\prime}=Y_{Z^{+}}^{\prime}=Y_{e^{-}}^{\prime}=0$ at $x \rightarrow \infty$.

The solution of the problem provides the eigenvalue

$\mu=\frac{\rho A_{I I} D_{T}}{S_{L}^{2} W}$

which determines completely the flame velocity $S_{L}$. Also, the following non-dimensional parameters appear in the above formulation: the Zeldovich number $\beta=10$, the dimensionless heat of reaction $Q=q_{I I} Y_{F_{0}} /\left[c_{p}\left(T_{c}-T_{0}\right) W_{F}\right]$, with $q_{I I}$ the total heat released from reactions II, the Lewis numbers of fuel $L e_{F}=D_{T} / D_{F}$ and radical $L e_{Z}=D_{T} / D_{Z}$ and the heat release parameter $\gamma=\left(T_{c}-T_{0}\right) / T_{c}=0.7$.

The non-dimensional reaction rates are written as

$\omega_{I}=\beta^{2} Y_{Z} Y_{F} \exp \left\{\beta \frac{\theta-1}{1+\gamma(\theta-1)}\right\}$
$\omega_{I I}=Y_{Z}$

$\omega_{I I I}=\beta^{2} \mathcal{A} Y_{Z} \exp \left\{(\beta+\Delta \beta) \frac{\theta-1}{1+\gamma(\theta-1)}\right\}$

$\omega_{I V}=\mathcal{B} Y_{Z^{+}} Y_{e^{-}}$

with $\mathcal{A}=\frac{A_{I I I}}{A_{I}} \frac{W_{Z^{+}} W_{F}}{W_{Z} \bar{W}} \frac{e^{-\Delta \beta / \gamma}}{Y_{F_{0}}}, \mathcal{B}=\frac{A_{I V}}{A_{I I}} \frac{\bar{W} W_{Z}}{W_{Z^{+}} W_{F}} Y_{F_{0}}$ and $\Delta \beta=\gamma \frac{E_{I I I}-E_{I}}{R_{g} T_{c}}$ representing the effect of a differen-

tial activation energy between the chain-branching and the chemiionization steps. In real flames, the heat released through the termination reaction $q_{I I}$ is different to that released through the dissociative recombination $q_{I V} \neq q_{I I}$. Nevertheless, and for the sake of simplicity, we will assume hereafter that $q_{I V}=q_{I I}$. In this case, the system of equations described above in (4)-(7) admits a first integral that allows the calculation of the temperature downstream of the reaction region $\theta_{\infty}=Q\left(1-F_{\infty}\right)$, where $F_{\infty}$ is the fuel leakage at $x \rightarrow \infty$, facilitating the physical interpretation of the parameter $Q$. Notice that if $q_{I V} / q_{I I} \neq 1$ the maximum flame temperature is diminished due to the reduction of the radical $Z$ that is consumed through reaction II, and the maximum flame temperature will be given by $\theta_{\infty}=Q\left[1-F_{\infty}-\right.$ $\left.\mu \mathcal{B}\left(1-q_{I V} / q_{I I}\right) \int_{-\infty}^{\infty} Y_{Z^{+}} Y_{e^{-}} d x\right]$.

The spatial distribution of non-dimensional electric field

$E=E^{\prime} /\left[v^{+}\left(\rho D_{T} A_{I I} / W\right)^{1 / 2}\right]$ depends on the spatial distribution of the charged species and is given by

$\frac{d E}{d x}=\frac{\mu^{1 / 2}}{\varepsilon}\left(Y_{Z^{+}}-Y_{e^{-}}\right) \quad E(x \rightarrow-\infty)=E_{0}$

with $\varepsilon=v^{+}\left(\varepsilon_{0} / e\right)\left(A_{I I} W_{Z^{+}}\right) /\left(W Y_{F_{0}} W_{Z} / W_{F}\right)$ and $E_{0}$ the external electric field applied.

The large electrons mobility anticipates an effective diffusion of the electrons away from the flame. In order to satisfy the boundary conditions specified above, the limits of the computational domain must reach distances of the order $|x| \sim\left(\mathrm{m}^{+} / \mathrm{m}^{-}\right) \gg 1$. Nevertheless, an asymptotic approximation at $x \rightarrow-\infty$ of $Y_{e^{-}}$and the associated induced electric field $E$ can be derived from Eqs. (7) and (13) by imposing $Y_{Z^{+}}=w_{I I I}=w_{I V}=0$ to give

$1-\mu^{1 / 2}\left(m^{+} / m^{-}\right)^{1 / 2} E=\frac{\left(m^{+} / m^{-}\right)^{1 / 2}}{L e_{Z^{+}}} Y_{e^{-}}^{-1} \frac{\partial Y_{e^{-}}}{\partial x}$

with

$1-\mu^{1 / 2}\left(m^{+} / m^{-}\right)^{1 / 2}$

$E=\left(\left(1-\mu^{1 / 2}\left(m^{+} / m^{-}\right)^{1 / 2} E_{0}\right)^{2}-2 \frac{\mu\left(m^{+} / m^{-}\right)}{\varepsilon L e_{Z^{+}}} Y_{e^{-}}\right)^{1 / 2}$

The straightforward integration of (14) gives the asymptotic behavior of the electrons mass fraction 
$Y_{e^{-}}=\frac{a^{2}}{b}\left[1-\tanh ^{2}\left\{-\frac{L e_{z} a}{\left(m^{+} / m^{-}\right)}(x+\mathcal{C})\right\}\right]$

$a=1-\mu^{1 / 2}\left(m^{+} / m^{-}\right)^{1 / 2} E_{0}$

$b=2 \frac{\mu\left(m^{+} / m^{-}\right)}{\varepsilon L e_{z}}$,

to be used as a substitute of the boundary condition at $x \rightarrow-\infty$ given above.

\section{Numerical method}

The problem defined by Eqs. (3)-(7) with the corresponding boundary conditions, was solved numerically to compute the eigenvalue $\mu$ and the profiles of temperature and species in a non-uniform grid spanning from $x_{\min }=-800$ to $x_{\max }=200$ with a maximum clustering of points around the flame location $x=0$. The spatial derivatives were discretized using second order, threepoint central differences in a grid formed by $N=5000$ points, what gives a minimum spacing $d x \simeq 0.02$ at $x=0$. A $50 \%$ increase in the number of points was used in some cases to test the grid independence of the numerical solution.

The eigenvalue $\mu$ and the profiles of temperature and species were computed using an iterative method based on a GaussSeidel procedure with over-relaxation that takes advantage of the invariance of the equations to a translation in the coordinate $x$. Using this property, a random value of temperature $\theta^{*}$ is forced at the grid point $x^{*}$ such that $\theta\left(x^{*}\right)=\theta^{*}$, what gives an additional condition that allows the calculation of the eigenvalue $\mu^{k}$ at the iteration $k$ from Eq. (3). To avoid the divergence of the method, we used a relaxation parameter $\alpha$ so that the value of the eigenvalue used at the next iteration $k+1$ is given by $\mu^{k+1}=\alpha \mu^{k}+(1-\alpha) \mu^{k-1}$. Typical values of above mentioned parameters are $\alpha=0.5$ and $\theta^{*}=0.65$. A comprehensive description of the numerical procedure outlined above can be found in [17].

\section{Results}

\subsection{Structure}

We begin by examining the basic structure of flames modeled in this formulation as shown in Fig. 1. The solutions for the neutral species $Y_{F}, Y_{Z}$ and $\omega_{I}$ closely follow those found in prior work studying reactions I and II [6]. $\theta$ initially follows the neutral solution, but develops more slowly in the later stages of the flame. This follows naturally considering that, with small values of $\mathcal{A}$ and $\Delta \beta \geqslant 0$, reactions I and II are dominant until $Y_{F}$ becomes small and temperature overcomes the higher activation energy in reaction III.

Once reaction III begins to proceed in earnest, heat release becomes highly dependent upon the presence of both $Y_{Z^{+}}$and $Y_{e^{-}}$. Upon the application of a positive $E_{0}$ we see significant shifts in the profiles of both ion species. Electron concentration before and within the flame region is elevated, permitting earlier electron-proton recombination. The resulting accelerated heat evolution is subtle but present in $\theta$ and, due to exponential temperature dependence, raises the
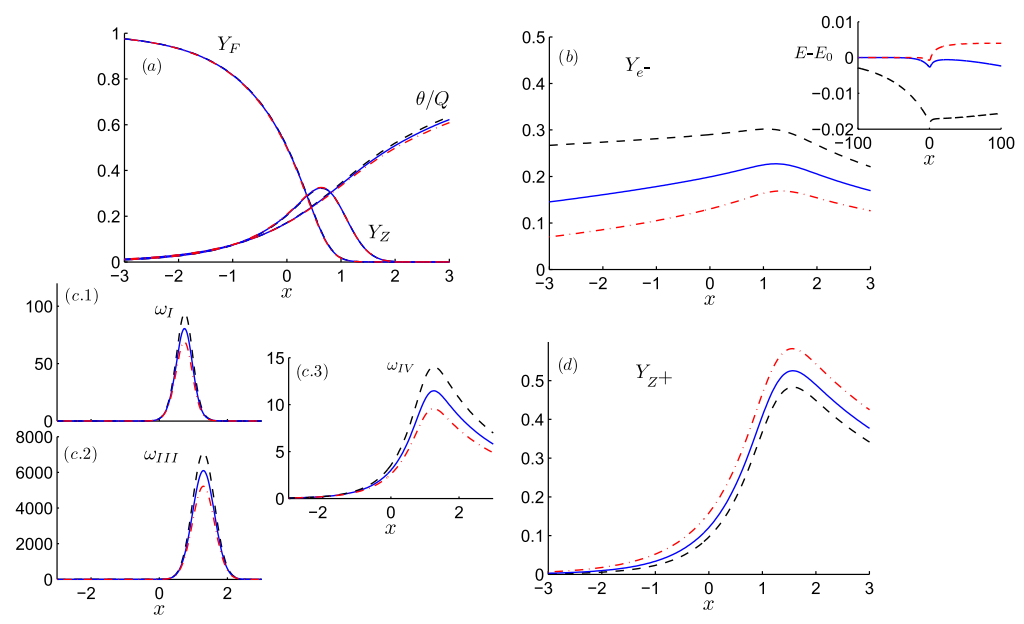

Fig. 1. (a) Profiles of fuel $Y_{F}$, radical $Y_{Z}$ and temperature $\theta / Q$, (b) and (d) Electron and proton mass fraction profiles, (c.1) chain-branching reaction rate $\omega_{I}$, (c.2) proton-electron production rate $\omega_{I I I}$ and (c.3) proton-electron recombination reaction rate $\omega_{I V}$ for $E_{0}=0$ (blue, solid line), $E_{0}=0.65$ (black, dashed line) and $E=-0.65$ (red dotdashed lines) with $\beta=10, \Delta \beta=1, \mathcal{A}=0.1, \mathcal{B}=100, \varepsilon=100, \mathrm{~m}^{+} / \mathrm{m}^{-}=100$ and $Q=5$. The inset of Fig. $1(\mathrm{~b})$ represents the auto-induced electric field created by the charge displacement near the flame. (For interpretation of the references to colour in this figure legend, the reader is referred to the web version of this article.) 


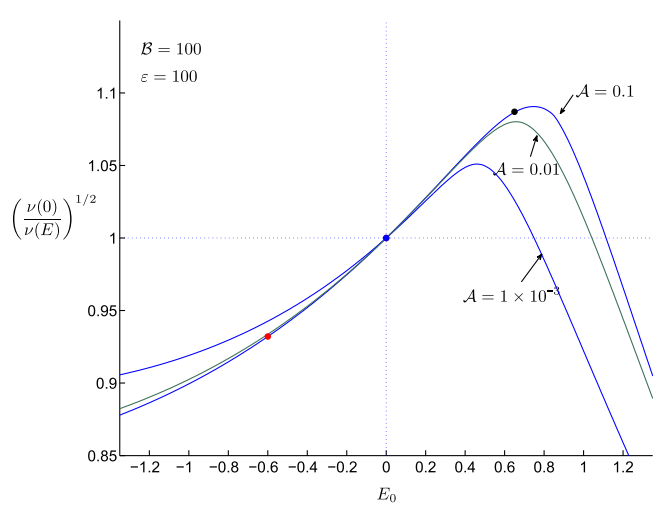

Fig. 2. Influence of $\mathcal{A}$ on Flame speed vs. electric field $E_{0}$ for $\beta=10, \Delta \beta=1, \varepsilon=100, \mathcal{B}=100, Q=5$ $m^{+} / m^{-}=100$. Points for $\mathcal{A}=0.1$ correspond to the cases in Fig. 1.

peak values of $\omega_{I}$ and $\omega_{I I I}$. So, we find that by controlling the delayed heat release of the chemiionization path it is possible to realize significant changes in total reaction rate, which is to say, flame speed as is found in Fig. 2. The opposite effect is observed when a negative electric field is applied. Recombination is delayed, temperature rises more slowly, reaction rates are decreased and flames speed decreases.

Observation of ion species only in the flame region is not sufficient to fully account for the processes at work. Particularly, a wider field of view is necessary to understand the local rise and fall of $Y_{e^{-}}$. The inset of Fig. 1(b) plots the self-induced electric field, from which the net charge may be deduced, over a large domain. In the case where $E_{0} \geqslant 0$, we see that $\partial E / \partial x \leqslant 0$ for $x \leqslant 0$ while $\partial E / \partial x>0$ (weakly) for $x>0$ (weakly), indicating that electrons are shifted strongly into both the flame sheet and pre-flame regions. This accumulation increases the availability of electrons within the flame sheet to participate in reaction IV and release heat $q_{I V}$ closer to the unreacted fuel. Where $E_{0} \leqslant 0$, electrons are driven far into the post-flame region. This effectively robs the flame sheet of a fraction of $Q$ by separating the components, $Y_{Z^{+}}$ and $Y_{e^{-}}$necessary for reaction IV.

Fundamentally, their high mobility causes electrons to be a deficient or limiting component for flame propagation. The influence of $E_{0}$ and the reason for its direction dependence come from a capacity to oppose or enhance the loss of electrons by advection and diffusion. Electrons driven forward by positive $E_{0}$ are simply not lost. They accumulate ahead of the flame but are not destroyed and remain available to react within the flame sheet. Electrons driven behind the flame, conversely, quickly become so far removed from the flame sheet that the heat they release cannot contribute meaningfully to propagation.

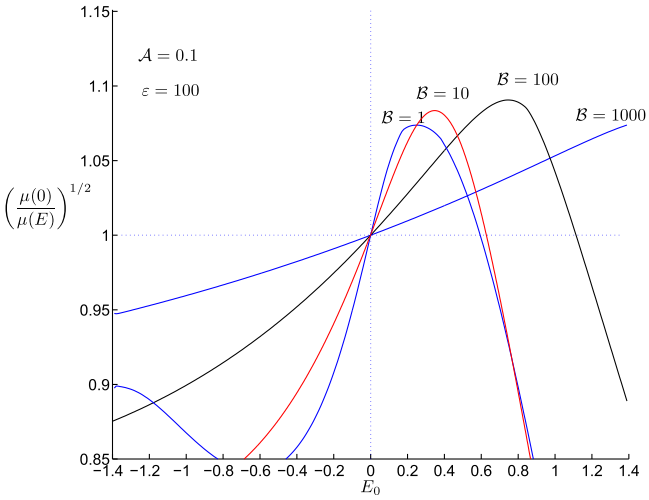

Fig. 3. Influence of $\mathcal{B}$ on Flame speed vs. electric field $E_{0} \quad$ for $\quad \beta=10, \Delta \beta=1, \varepsilon=100, \mathcal{A}=0.1, Q=5$, $m^{+} / m^{-}=100$

Note that flame speed will not increase without limit as $E_{0}$ increases. As seen in Figs. 2-5, for each set of parameters $(\mathcal{A}, \mathcal{B}$, etc.) there is a critical value of $E_{0}$ at which flame speed is maximal and further increases in $E_{0}$ reduce flame speed.

\subsection{Parameters}

In the interest of generality, the dimensionless parameters $\mathcal{A}, \mathcal{B}, \varepsilon$ and $Q$ have been kept somewhat arbitrary, but their values do modify the sensitivity of flames to externally applied electric fields.

First, we consider $\mathcal{A}$ which controls the rate at which $Y_{Z}$ produces both $Y_{Z^{+}}$and $Y_{e^{-}}$. Increasing the value of $\mathcal{A}$ increases both the intensity of the enhancement effect and the critical value of $E_{0}$. The first point is unsurprising given that $\mathcal{A}$ increases the production of species directly influenced by $E_{0}$. The second stems from increased

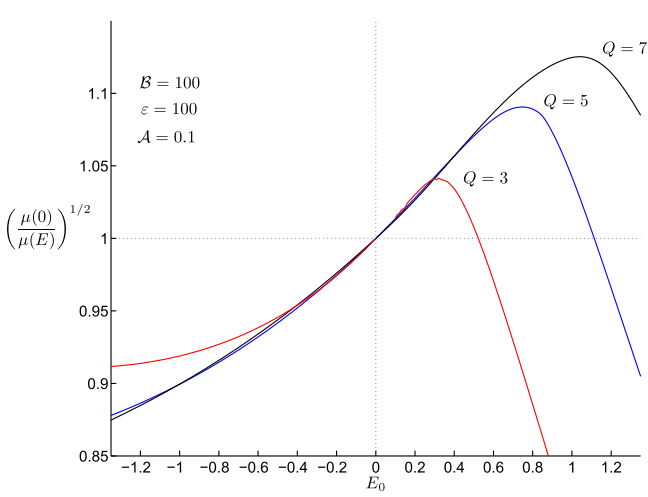

Fig. 4. Influence of $O$ on Flame speed vs. electric field $E_{0}$ for $\beta=10, \Delta \beta=1, \varepsilon=100, \mathcal{A}=0.1, \mathcal{B}=100$, $m^{+} / m^{-}=100$ 


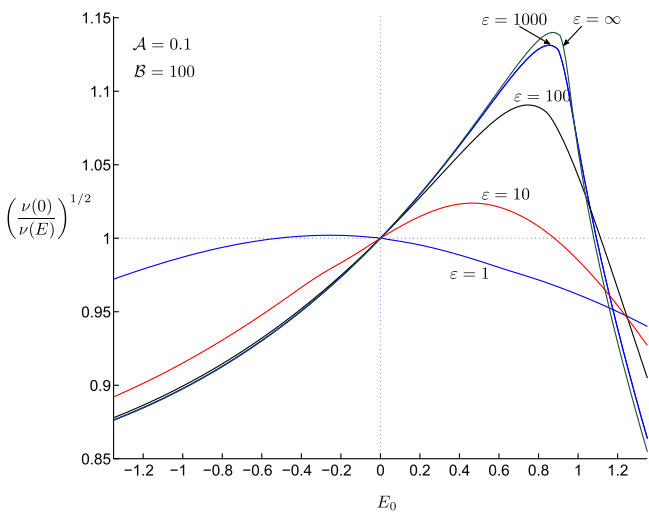

Fig. 5. Influence of $\varepsilon$ on flame speed vs. electric field $E_{0}$ for $\mathcal{B}=100, \mathcal{A}=0.1, Q=5, \mathrm{~m}^{+} / \mathrm{m}^{-}=100$.

self-induced electric fields made possible by higher total concentrations of both ion species. These self-induced fields naturally oppose the charge separation effect that limits flame speed enhancement.

The efficiency with which the ion species recombine is governed by $\mathcal{B}$. In the extreme that $\mathcal{B} \rightarrow \infty$ the recombination would occur instantly, with the release of $q_{I V}$ being limited by reaction III. Figure 3 shows that as this $\mathcal{B}$ is increased, ion transport diminishes and higher electric fields are necessary to achieve similar increases in flame speed. The maximum value of $\mu(0) / \mu(E)^{1 / 2}$ increases very weakly with $\mathcal{B}$. High rates of recombination can only shift the peak of $\omega_{I V}$ as far forward as that of $\omega_{I I I}$, which limits the potential for propagation enhancement.

As consistent with laminar flame theory and evidenced by prior work on laminar flames $[6,17]$, increases in the heat release parameter $Q$ can dramatically increase flame speed. We see in Fig. 4 that this does not change the initial value of $\partial(\mu(0) / \mu(E))^{1 / 2} / \partial E$, but does increase the critical value of $E_{0}$ and, therefore, the maximum value of $(\mu(0) / \mu(E))^{1 / 2}$. We have discussed the role of self-induced electrostatic fields in opposing detrimental charge separation, but the constant forward travel of the flame front also serves minimize the relative motion of ions when $E_{0}>0$. More simply, one may consider this as a balance between flame speed, $S_{L}$, and the electromotive component of drift velocity in Eq. (1).

Lastly, observe in Fig. 5 the impact of $\varepsilon$. Recalling, of course, from Eq. (13) that intensity of the auto-induced field will vary inversely with ع. $\varepsilon=\infty$ corresponds to ions which are acted upon solely by $E_{0}$ and diffusion. As such, flame speed exhibits its strong sensitivity to $E_{0}$.

For small values, of $\varepsilon$, a different phenomenon develops. When $\varepsilon \approx 1$, any charge separation is vigorously opposed and transport of $Y_{Z^{+}}$by the imposed field can shift the heat release by reaction IV. Hence we see a reversal in the response of flame speed. A positive $E_{0}>0$ tends to drive positive ions away from the flame front, delaying heat release. Similarly, weakly negative values $E_{0}$ will actually marginally increase flame speeds.

\section{Conclusions}

The effect of an external electric field on a freely propagating, planar and adiabatic premixed flame is investigated for an idealized chemical mechanism that includes a two-step, chainbranching model and two additional reactions to account for the production and consumption of charged species.

The basic structure of the flames modeled in this paper follows the structure described by previous studies [5] in the low temperature region of the flame. Once $Y_{F} \ll 1$, the temperaturedependent chemiionization step becomes dominant and the heat released is then controlled by the concentration of protons and electrons, as indicated by reaction IV.

The application of an external electric field $E_{0}$ changes the distribution of protons and electrons around the thin chain-branching layer what, in turn, modifies the rate at which the heat is released and induces changes in the flame speed. Concretely, the application of a positive electric field $E_{0}$ promotes the accumulation of electrons in the cold region of the flame and increases its availability to react with the protons through reaction IV once they temperature is sufficiently high to overcome the activation energy of reaction III. The opposite effect is observed when $E_{0}<0$.

The sensitivity of the flame velocity regarding several of the non-dimensional parameters of the problem have been tested. Specifically, we focused on the effect of the frequency factors of reaction III and IV, $\mathcal{A}$ and $\mathcal{B}$ respectively, the heat released parameter $Q$ and the non-dimensional permittivity $\bar{\varepsilon}$. For all the four parameters, the calculations revealed a decrease of the flame speed for $E_{0}<0$, due to the reduction of the electrons concentration before the flame. On the other hand, an increase of the flame speed is observed for $E_{0, \max }>E_{0}>0$, where $E_{0, \max }$ is the maximum electric field at which $(\mu(0) / \mu(E))^{1 / 2}>1$. For values of $E_{0}>E_{0, \max }$, the effect of the electric field on the flame speed is reverted and $(\mu(0) / \mu(E))^{1 / 2}<1$. The reason for this is the effective diffusion of electrons towards the cold size of the flame induced by the electric field, what reduces the rate of the dissociative recombination reaction and delays the rise of temperature behind the flame.

Maximum flame speed increments of around $15 \%$ have been found for a specific combination of the non-dimensional parameters of the 
problem. This number is modest when compared with the most extreme experimental data found in the literature [1], but agrees with the saturation effect reported in [18] for lean flames. The model presented is not a tool for quantitative prediction, but a tool for developing an improved qualitative understanding of the phenomenon. This can serve as a basis for progressively building less abstract models by including additional features, such as thermal expansion or a more complex chemical kinetics.

\section{Acknowledgements}

This collaborative research was supported by the Spanish MCINN under Project \#ENE201233213 and by King Abdullah University of Science and Technology (KAUST), Cooperative Agreement \# 025478 entitled, Electromagnetically Enhanced Combustion: Electric Flames.

\section{References}

[1] C.F.-P.D.C. Murphy, G. Noel, Electric Field Effects on Premixed Methane-Air Flames in MillimeterScale Channels, Taipei, Taiwan, 2013.

[2] Y.B. Zel'dovich, Zh. Phys. Khim. 22 (1948) 27-49.

[3] I. Zel'dovich, G.I. Barenblatt, V. Librovich, G. Makhviladze, Mathematical theory of combustion and explosions, Consultants Bureau, New York, NY, 1985.

[4] A. Liñán, A Theoretical Analysis of Premixed Flame Propagation with an Isothermal Chain Reaction, Defense Technical Information Center, 1971

[5] J. Dold, R. Thatcher, A. Omon-Arancibia, J. Redman, Proc. Combust. Inst. 29 (2002) 1519-1526.

[6] J. Dold, Combust. Theory Model. 11 (2007) 909948.

[7] G.J. Sharpe, SIAM J. Appl. Math. 70 (2009) 866884.

[8] T. Pedersen, R.C. Brown, Combust. Flame 94 (1993) 433-448.

[9] H. CalcoteProc. Combust. Inst., 8, 1962, pp. 184 199.

[10] H. Calcote, S. Kurzius, W. MillerProc. Combust. Inst., 10, 1965, pp. 605-619.

[11] M. Papac, D. Dunn-Rankin, Combust. Theory Model. 12 (2007) 23-44.

[12] M. Belhi, P. Domingo, P. Vervisch, Combust. Flame 157 (2010) 2286-2297.

[13] J. Cancian, B. Bennett, M. Colket, M. Smooke, Combust Theory Model 17 (2013) 294-315.

[14] R.F. Probstein, Physicochemical Hydrodynamics: An Introduction, Wiley.com, 1995.

[15] A.B. Fialkov, Prog. Energy Combust. Sci. 23 (1997) 399-528.

[16] J.-L. Delcroix, Physique des Plasmas, DUNOD, 1963.

[17] V.N. Kurdyumov, D. Fernández-Galisteo, Combust. Flame 159 (2012) 3110-3118.

[18] H. Jaggers, A. VonEngel, Combust. Flame 16 (1971) 275-285. 